\title{
Supply Chain Contracts with Multiple Retailers in a Fuzzy Demand Environment
}

\author{
Shengju Sang \\ Department of Economics, Heze University, Heze 274015, China \\ Correspondence should be addressed to Shengju Sang; sangshengju@163.com
}

Received 17 March 2013; Accepted 11 July 2013

Academic Editor: Daoyi Dong

Copyright (C) 2013 Shengju Sang. This is an open access article distributed under the Creative Commons Attribution License, which permits unrestricted use, distribution, and reproduction in any medium, provided the original work is properly cited.

This study investigates supply chain contracts with a supplier and multiple competing retailers in a fuzzy demand environment. The market demand is considered as a positive triangular fuzzy number. The models of centralized decision, return contract, and revenue-sharing contract are built by the method of fuzzy cut sets theory, and their optimal policies are also proposed. Finally, an example is given to illustrate and validate the models and conclusions. It is shown that the optimal total order quantity of the retailers fluctuates at the center of the fuzzy demand. With the rise of the number of retailers, the optimal order quantity and the fuzzy expected profit for each retailer will decrease, and the fuzzy expected profit for supplier will increase.

\section{Introduction}

Over the last decade or so, supply chain management has emerged as a key area of research among the practitioners of operations research. In recent years, coordination mechanism of supply chain contracts has become one of the most challenging issues facing both practitioners and scholars. Supply chain contracts such as return contract and revenuesharing contract are instruments for supply chain coordination, which shift the uncertain demand from the retailer to the supplier, thus encouraging the retailer to increase order quantities.

A large body of literature has explored to coordinate the supply chain with return contract and revenue-sharing contract during the last two decades. Pasternack [1] first claimed that an appropriate return policy can fully coordinate a single-supplier single-retailer supply chain, which was then extended by Mantrala and Raman [2] to the situation where the retailer had several stores. Taylor [3] and Lee et al. [4] studied the return contract with effort-dependant demand. They showed that in this problem, it attained supply chain coordination combined with feedback policy. Yao et al. [5] analyzed the profits of both actors when the manufacturer and retailer shared or did not share the forecast information in returns policy. Yue and Raghunathan [6] discussed the impact of a full return policy as well as information sharing on the manufacturer and the retailer under information asymmetry. Bose and Anand [7] considered the wholesale price as an exogenous price to study returns policies for coordinating the supply chain. They showed that, in general, an equilibrium returns policy was not Pareto efficient with respect to a price-only contract, but when the wholesale price was sufficiently high, the equilibrium returns policy was Pareto efficient. These conclusions were consistent with those of Yao et al. [8]. Ding and Chen [9] studied the return contract issues of a three-level supply in a single-period model. Yao et al. [10] analyzed the impact of price-sensitivity factors on characteristics of return contract in a single-period product supply chain. Mollenkopf et al. [11] used an empirical study to explore how internet product returns management systems affect loyalty intentions. Chen and Bell $[12,13]$ showed that the customer returns affect the firm's pricing and inventory decision and proposed an agreement between the manufacturer and the retailer that includes two buyback prices. Chen [14] proposed a returns policy with a wholesale-pricediscount scheme that can achieve supply chain coordination. Ai et al. [15] analyzed the implementation of full returns policies in the chain-to-chain competition. 
Revenue-sharing contract has been applied in the video cassette rental and movie industry with much success. Giannoccaro and Pontrandolfo [16] showed that revenue-sharing could coordinate members in the newsboy channel with three stages: supplier, manufacturer, and retailer. Cachon and Lariviere [17] intensively discussed a revenue-sharing contract between a single supplier and a single retailer in a singleperiod newsboy problem. Gupta and Weerawat [18] designed a revenue-sharing contract to maximize the centralized revenue by choosing an appropriate inventory level. Yao et al. [19] investigated a revenue-sharing contract for coordinating a supply chain comprising one manufacturer and two competing retailers. Linh and Hong [20] studied a revenue-sharing contract in a two-period newsboy problem. Van Der Rhee et al. [21] proposed a revenue-sharing mechanism in multiechelon supply chains. Ouardighi and Kim [22] considered a single supplier collaborating with two manufacturers on designing quality improvements for their respective products under a revenue-sharing contract. Krishnan and Winter [23] studied the role of revenue-sharing contracts in supply chains and established a foundation in aligning incentives. Sheu [24] explored revenue-sharing contracts under price promotion to end customers with three types of promotional demand patterns. Zhang et al. [25] investigated a revenuesharing contract with demand disruptions in a supply chain comprising one manufacturer and two competing retailers. Palsule-Desai [26] studied revenue-dependent contracts and revenue-independent contracts in a two-period model, and they showed that both types of revenue-sharing contracts could coordinate the supply chain; however, there existed situations in which revenue-dependent contracts outperformed revenue-independent contracts. The conventional studies have focused on the cases in which the demands are probabilistic. In other words, the demands follow certain distribution function. However, in practice, especially for new products, the probabilities are not known due to lack of history data. In this case the demands are suitably described subjectively by linguistic terms, such as "high," "low," or "approximately equal $d$, but definitely not less than $a$ and not greater than $b$." Thus, the uncertain theory, rather than the traditional probability theory, is well suited to the supply chain models problem. Therefore, we assume that the external demand can be approximately forecasted and expressed as a triangular membership function.

In this paper, the demands are approximately estimated by experts and regarded as fuzzy numbers. Return contract and revenue-sharing contract with multiple competing retailers in a fuzzy demand environment will be discussed, and the impact of the supplier's production cost and the number of retailers on the models will be analyzed.

The rest of the paper is organized as follows. Section 2 introduces some definitions and propositions about fuzzy set theory and notations related to this paper. Section 3 develops three fuzzy supply models with multiple competing retailers. Section 4 provides a numerical example to illustrate the result of the proposed contracts. Section 5 summarizes the work done in this paper.

\section{Preliminaries}

\subsection{Fuzzy Set Theory}

Definition 1. The fuzzy set $\widetilde{A}=\left(a_{1}, a_{2}, a_{3}\right)$, where $a_{1}<a_{2}<a_{3}$ and defined on $R$, is called the triangular fuzzy number, if the membership function of $\widetilde{A}$ is given by

$$
\mu_{\widetilde{A}}(x)= \begin{cases}\frac{x-a_{1}}{a_{2}-a_{1}}, & \text { if } a_{1} \leq x \leq a_{2}, \\ \frac{a_{3}-x}{a_{3}-a_{2}}, & \text { if } a_{2}<x \leq a_{3}, \\ 0, & x \notin\left(a_{1}, a_{3}\right),\end{cases}
$$

where $a_{1}$ and $a_{3}$ are the lower limit and upper limit, respectively, of the triangular fuzzy number $\widetilde{A}$. For $x \in\left[a_{1}, a_{2}\right]$, the left membership function $\widetilde{A}_{L}(x)=\left(x-a_{1}\right) /\left(a_{2}-a_{1}\right)$ is an increase function of $x$. For $x \in\left(a_{2}, a_{3}\right]$, the right membership function $\widetilde{A}_{R}(x)=\left(a_{3}-x\right) /\left(a_{3}-a_{2}\right)$ is a decrease function of $x$.

Definition 2. The triangular fuzzy number $\widetilde{A}$ is called the positive triangular fuzzy number if $a_{1}>0$.

Definition 3. For any $\alpha \in[0,1]$, the set $\widetilde{A}(\alpha)=\left\{x \mid \mu_{\widetilde{A}}(x) \geq\right.$ $\alpha\}$ is called the $\alpha$ cut set of $\widetilde{A}$. $\widetilde{A}(\alpha)$ is a nonempty bounded closed interval contained in the set of real numbers, and it can be denoted by

$$
\widetilde{A}(\alpha)=\left[\widetilde{A}_{L}^{-1}(\alpha), \widetilde{A}_{R}^{-1}(\alpha)\right],
$$

where $\widetilde{A}_{L}^{-1}(\alpha)$ and $\widetilde{A}_{R}^{-1}(\alpha)$ are, respectively, the left and right boundaries of $\widetilde{A}(\alpha)$, with

$$
\begin{aligned}
& \widetilde{A}_{L}^{-1}(\alpha)=\inf \left\{x \in R: \mu_{\widetilde{A}(x)} \geq \alpha\right\}, \\
& \widetilde{A}_{R}^{-1}(\alpha)=\sup \left\{x \in R: \mu_{\widetilde{A}(x)} \geq \alpha\right\} .
\end{aligned}
$$

Example 4. For any $\alpha \in[0,1]$, the $\alpha$ cut set of a triangular fuzzy number $\widetilde{A}=\left(a_{1}, a_{2}, a_{3}\right)$ is

$$
\begin{aligned}
& \widetilde{A}_{L}^{-1}(\alpha)=a_{1}+\left(a_{2}-a_{1}\right) \alpha, \\
& \widetilde{A}_{R}^{-1}(\alpha)=a_{3}-\left(a_{3}-a_{2}\right) \alpha .
\end{aligned}
$$

Based on the extension principle in fuzzy sets, we have the following Propositions 5 and 6.

Proposition 5. For any $\alpha \in[0,1]$, let $\widetilde{A}$ be a positive triangular fuzzy number and let $k$ be a nonzero real number; then

$$
k \widetilde{A}(\alpha)= \begin{cases}{\left[k \widetilde{A}_{L}^{-1}(\alpha), k \widetilde{A}_{R}^{-1}(\alpha)\right],} & k \in R^{+}, \\ {\left[k \widetilde{A}_{R}^{-1}(\alpha), k \widetilde{A}_{L}^{-1}(\alpha)\right],} & k \in R^{-} .\end{cases}
$$

Proposition 6. For any $\alpha \in[0,1]$, let $\widetilde{B}(\alpha)=\left[\widetilde{B}_{L}^{-1}(\alpha), \widetilde{B}_{R}^{-1}(\alpha)\right]$ and $\widetilde{C}(\alpha)=\left[\widetilde{C}_{L}^{-1}(\alpha), \widetilde{C}_{R}^{-1}(\alpha)\right]$, respectively, be the $\alpha$ cut set of the positive triangular fuzzy numbers $\widetilde{B}$ and $\widetilde{C}$; then

$$
\begin{aligned}
& \widetilde{B}(\alpha)+\widetilde{C}(\alpha)=\left[\widetilde{B}_{L}^{-1}(\alpha)+\widetilde{C}_{L}^{-1}(\alpha), \widetilde{B}_{R}^{-1}(\alpha)+\widetilde{C}_{R}^{-1}(\alpha)\right], \\
& \widetilde{B}(\alpha)-\widetilde{C}(\alpha)=\left[\widetilde{B}_{L}^{-1}(\alpha)-\widetilde{C}_{R}^{-1}(\alpha), \widetilde{B}_{R}^{-1}(\alpha)-\widetilde{C}_{L}^{-1}(\alpha)\right] .
\end{aligned}
$$


Proposition 7 (see B. Liu and Y.-K. Liu [27]). Let $\widetilde{A}$ be a positive triangular number; the expected value of $\widetilde{A}$ is

$$
E[\widetilde{A}]=\frac{1}{2} \int_{0}^{1}\left[\widetilde{A}_{L}^{-1}(\alpha)+\widetilde{A}_{R}^{-1}(\alpha)\right] \mathrm{d} \alpha .
$$

Proposition 8 (see Y.-K. Liu and B. Liu [28]). Let $\widetilde{A}$ and $\widetilde{B}$ be two independent positive triangular fuzzy numbers with finite expected values. Then for any real numbers $a$ and $b$, one has

$$
E[a \widetilde{A}+b \widetilde{B}]=a E[\widetilde{A}]+b E[\widetilde{B}] .
$$

2.2. Problem Descriptions. Consider a single-period setting for a two-echelon supply, consisting of a supplier and multiple competing retailers with fuzzy demand. We assume that at the beginning of the selling season, the retailer $i(i=1,2, \ldots, n)$ has no inventory on hand and must decide the order quantity $q_{i}$ from the supplier. Then, the retailer $i$ sells his order of short-life products, such as personal computers, consumer electronics, or fashion items, with high uncertain demand. The products are sold only in one period. As the lead times of such goods are much longer than their selling season, the actors have no chance to place a second order.

We consider the total uncertain demand faced by the retailers as a positive triangular fuzzy variable $\widetilde{D}=\left(d_{1}, d_{2}, d_{3}\right)$ with the most possible value $d_{2}$, where $0<d_{1}<d_{2}<d_{3}$. The fuzzy demand $\widetilde{D}$ means that the total demand is about $d_{2}$. $d_{1}$ and $d_{3}$ are the lower limit and upper limit, respectively, of the fuzzy demand $\widetilde{D}$ and described by a general membership function $\mu_{\widetilde{D}}(x)$ :

$$
\mu_{\widetilde{D}}(x)= \begin{cases}L(x), & x \in\left[d_{1}, d_{2}\right], \\ R(x), & x \in\left(d_{2}, d_{3}\right], \\ 0, & x \notin\left(d_{1}, d_{3}\right) .\end{cases}
$$

Let the total retail demand be divided between the $n$ retailers proportional to their stocking quantity; that is, retailer $i$ 's demand, $\widetilde{D}_{i}$, is

$$
\widetilde{D}_{i}=\left(\frac{q_{i}}{q}\right) \widetilde{D},
$$

where $q=\sum_{i=1}^{n} q_{i}$.

The following notations are used for a product in the models:

$p$ : the retail price;

$w$ : the wholesale price;

$c$ : the per unit product cost incurred to the supplier;

$b$ : the return price offered by supplier in return contract;

$\Phi$ : the fraction revenue of the retailer in revenue-

sharing contract and $0<\Phi<1$;

$\widetilde{\Pi}_{S}$ : the fuzzy profit of the supplier;

$\widetilde{\Pi}_{R_{i}}$ : the fuzzy profit of the retailer $i$;

$\widetilde{\Pi}_{S C}$ : the fuzzy profit of the supply chain.

The supplier and the retailer $i$ are assumed to be risk neutral and pursued maximization of their fuzzy expected profits.

\section{Model Analysis}

3.1. Centralized Decision Making with Fuzzy Demand. Consider a supply chain occupied by an integrated actor, which can also be regarded as the retailers and the supplier-making cooperation. The fuzzy profit of two-stage supply chain can be expressed as

$$
\begin{aligned}
& \widetilde{\Pi}_{\mathrm{SC}}=p \\
& \min \{q, \widetilde{D}\}-c q \\
& \text { s.t. } d_{1} \leq q \leq d_{3} .
\end{aligned}
$$

Since the fuzzy demand $\widetilde{D}=\left(d_{1}, d_{2}, d_{3}\right)$ in (11) is a positive triangular fuzzy number, we know that the order quantity $q$ has two cases; that is, $q \in\left[d_{1}, d_{2}\right]$ or $q \in\left(d_{2}, d_{3}\right]$.

Theorem 9. When $c<p \leq 2 c$, the optimal total order quantity $q^{*}$ of the retailers is

$$
q^{*}=L^{-1}\left(\frac{2(p-c)}{p}\right) .
$$

Proof. If $q \in\left[d_{1}, d_{2}\right]$, then the $\alpha$ cut set of $\min \{q, \widetilde{D}\}$ is

$$
[\min \{q, \widetilde{D}\}](\alpha)= \begin{cases}{\left[L^{-1}(\alpha), q\right],} & \alpha \in[0, L(q)], \\ {[q, q],} & \alpha \in(L(q), 1] .\end{cases}
$$

(a) For $\alpha \in[0, L(q)]$, the $\alpha$ cut set of the supply chain's fuzzy profit is

$$
\left[\widetilde{\Pi}_{\mathrm{SC}}\right](\alpha)=\left[p L^{-1}(\alpha)-c q, p q-c q\right] .
$$

(b) For $\alpha \in(L(q), 1]$, the result turns to

$$
\left[\widetilde{\Pi}_{\mathrm{SC}}\right](\alpha)=[p q-c q, p q-c q]
$$

By (7), we can get the fuzzy expected profit $E\left[\widetilde{\Pi}_{\mathrm{SC}}\right]$ as

$$
\begin{aligned}
E\left[\widetilde{\Pi}_{\mathrm{SC}}\right]= & \frac{1}{2} \int_{0}^{L(q)}\left(p L^{-1}(\alpha)-c q+p q-c q\right) \mathrm{d} \alpha \\
& +\frac{1}{2} \int_{L(q)}^{1}(p q-c q+p q-c q) \mathrm{d} \alpha \\
= & \frac{1}{2} p\left(\int_{0}^{L(q)} L^{-1}(\alpha) \mathrm{d} \alpha-q L(q)+q\right)-c q .
\end{aligned}
$$

The first and second derivatives of $E\left[\widetilde{\Pi}_{\mathrm{SC}}\right]$ in (16) can be obtained as follows:

$$
\begin{gathered}
\frac{\mathrm{d} E\left[\widetilde{\Pi}_{\mathrm{SC}}\right]}{\mathrm{d} q}=\frac{1}{2} p(1-L(q))-c, \\
\frac{\mathrm{d}^{2} E\left[\widetilde{\Pi}_{\mathrm{SC}}\right]}{\mathrm{d} q^{2}}=-\frac{1}{2} p L^{\prime}(q) .
\end{gathered}
$$


Since $L(q)$ is an increasing function with $L^{\prime}(q)>0$, therefore $\mathrm{d}^{2} E\left[\widetilde{\Pi}_{\mathrm{SC}}\right] / \mathrm{d} q^{2}$ is negative and $E\left[\widetilde{\Pi}_{\mathrm{SC}}\right]$ is concave in $q$.

Hence, the optimal total order quantity of the retailers can be obtained by solving $\mathrm{d} E\left[\widetilde{\Pi}_{\mathrm{SC}}\right] / \mathrm{d} q=0$, which gives

$$
q^{*}=L^{-1}\left(\frac{2(p-c)}{p}\right) .
$$

Since $0<L\left(q^{*}\right) \leq 1$, thus we can get $c<p \leq 2 c$.

The proof of Theorem 9 is completed.

Theorem 10. When $p>2 c$, the optimal total order quantity $q^{*}$ of the retailers is

$$
q^{*}=R^{-1}\left(\frac{2 c}{p}\right)
$$

Proof. If $q \in\left(d_{2}, d_{3}\right]$, then the $\alpha$ cut set of $\min \{q, \widetilde{D}\}$ is

$$
[\min \{q, \widetilde{D}\}](\alpha)= \begin{cases}{\left[L^{-1}(\alpha), q\right],} & \alpha \in[0, R(q)], \\ {\left[L^{-1}(\alpha), R^{-1}(\alpha)\right],} & \alpha \in(R(q), 1] .\end{cases}
$$

(a) For $\alpha \in[0, R(q)]$, the $\alpha$ cut set of the supply chain's fuzzy profit is

$$
\left[\widetilde{\Pi}_{\mathrm{SC}}\right](\alpha)=\left[p L^{-1}(\alpha)-c q, p q-c q\right] .
$$

(b) For $\alpha \in(R(q), 1]$, the result turns to

$$
\left[\widetilde{\Pi}_{\mathrm{SC}}\right](\alpha)=\left[p L^{-1}(\alpha)-c q, p R^{-1}(\alpha)-c q\right] .
$$

By (7), we can get the fuzzy expected profit $E\left[\widetilde{\Pi}_{\mathrm{SC}}\right]$ as

$$
\begin{aligned}
E\left[\widetilde{\Pi}_{\mathrm{SC}}\right]= & \frac{1}{2} \int_{0}^{R(q)}\left(p L^{-1}(\alpha)-c q+p q-c q\right) \mathrm{d} \alpha \\
& +\frac{1}{2} \int_{R(q)}^{1}\left(p L^{-1}(\alpha)-c q+p R^{-1}(\alpha)-c q\right) \mathrm{d} \alpha \\
= & \frac{1}{2} p\left(\int_{R(q)}^{1} R^{-1}(\alpha) \mathrm{d} \alpha\right. \\
& \left.+q R(q)+\int_{0}^{1} L^{-1}(\alpha) \mathrm{d} \alpha\right)-c q .
\end{aligned}
$$

The first and second derivatives of $E\left[\widetilde{\Pi}_{\mathrm{SC}}\right]$ in (23) can be obtained as follows:

$$
\begin{aligned}
& \frac{\mathrm{d} E\left[\widetilde{\Pi}_{\mathrm{SC}}\right]}{d q}=\frac{1}{2} p R(q)-c, \\
& \frac{\mathrm{d}^{2} E\left[\widetilde{\Pi}_{\mathrm{SC}}\right]}{\mathrm{d} q^{2}}=\frac{1}{2} p R^{\prime}(q) .
\end{aligned}
$$

Since $R(q)$ is a decreasing function with $R^{\prime}(q)<0$, therefore $\mathrm{d}^{2} E\left[\widetilde{\Pi}_{\mathrm{SC}}\right] / \mathrm{d} q^{2}$ is negative and $E\left[\widetilde{\Pi}_{\mathrm{SC}}\right]$ is concave in $q$.

Hence, the optimal total order quantity of the retailers can be obtained by solving $\mathrm{d} E\left[\widetilde{\Pi}_{\mathrm{SC}}\right] / \mathrm{d} q=0$, which gives

$$
q^{*}=R^{-1}\left(\frac{2 c}{p}\right) .
$$

Since $0<R\left(q^{*}\right) \leq 1$, thus we can get $p>2 c$.

The poof of Theorem 10 is completed.

From (16) and (23), Theorems 9 and 10, we can easily obtain the optimal fuzzy expected value of the profit for the integrated supply chain, which is given by

$$
\begin{aligned}
& E\left[\widetilde{\Pi}_{\mathrm{SC}}\right]^{*} \\
& = \begin{cases}\frac{1}{2} p \int_{0}^{2(p-c) / p} L^{-1}(\lambda) \mathrm{d} \lambda, & c<p \leq 2 c, \\
\frac{1}{2} p\left(\int_{2 c / p}^{1} R^{-1}(\alpha) \mathrm{d} \lambda+\int_{0}^{1} L^{-1}(\alpha) \mathrm{d} \alpha\right), & p>2 c .\end{cases}
\end{aligned}
$$

3.2. Return Contract with Fuzzy Demand. In a return contract, the supplier sets a wholesale price $w$ and gives the retailer $i(i=1,2, \ldots, n)$ a return price $b$ for unsold products at the end of the season. The fuzzy profit of the retailer $i$ can be expressed as follows:

$$
\begin{aligned}
\widetilde{\Pi}_{R_{i}}\left(q_{i}, q_{-i}\right)= & p \min \left\{q_{i}, \widetilde{D}_{i}\right\}+b \max \left\{q_{i}-\widetilde{D}_{i}, 0\right\}-w q_{i} \\
= & p \min \left\{q_{i}, \frac{q_{i}}{q} \widetilde{D}\right\} \\
& +b \max \left\{q_{i}-\frac{q_{i}}{q} \widetilde{D}, 0\right\}-w q_{i},
\end{aligned}
$$

where $q=\sum_{i=1}^{n} q_{i}$ and $q_{-i}=q-q_{i}$.

The retailer $i(i=1,2, \ldots, n)$ tries to maximize its fuzzy expected profit $E\left[\widetilde{\Pi}_{R_{i}}\left(q_{i}, q_{-i}\right)\right]$ in return contract by choosing the optimal order quantity $q_{i}$, which solves the following model:

$$
\begin{array}{r}
E\left[\widetilde{\Pi}_{R_{i}}\left(q_{i}, q_{-i}\right)\right] \\
=E\left[p \min \left\{q_{i}, \frac{q_{i}}{q} \widetilde{D}\right\}+b \max \left\{q_{i}-\frac{q_{i}}{q} \widetilde{D}, 0\right\}-w q_{i}\right] \\
\text { s.t. } \frac{q_{i}}{q} d_{1} \leq q_{i} \leq \frac{q_{i}}{q} d_{3} .
\end{array}
$$

Theorem 11. When $c<p \leq 2 c$, the optimal wholesale price $w^{*}$ in return contract is

$$
\begin{aligned}
w^{*}=p-\frac{1}{2}(p-b)( & \frac{2(p-c)}{p}-\left(\frac{n-1}{n}\right) \\
\times & \left.\left(\frac{\int_{0}^{2(p-c) / p} L^{-1}(\alpha) \mathrm{d} \alpha}{L^{-1}(2(p-c) / p)}\right)\right) .
\end{aligned}
$$


Proof. If $q_{i} \in\left[\left(q_{i} / q\right) d_{1},\left(q_{i} / q\right) d_{2}\right]$, that is, $q \in\left[d_{1}, d_{2}\right]$, then the $\alpha$ cut sets of $\min \left\{q_{i},\left(q_{i} / q\right) \widetilde{D}\right\}$ and $\max \left\{q_{i}-\left(q_{i} / q\right) \widetilde{D}, 0\right\}$ are

$$
\begin{aligned}
& {\left[\min \left\{q_{i}, \frac{q_{i}}{q} \widetilde{D}\right\}\right](\alpha)} \\
& = \begin{cases}{\left[\frac{q_{i}}{q} L^{-1}(\alpha), q_{i}\right],} & \alpha \in(0, L(q)], \\
{\left[q_{i}, q_{i}\right],} & \alpha \in(L(q), 1],\end{cases} \\
& {\left[\max \left\{q_{i}-\frac{q_{i}}{q} \widetilde{D}, 0\right\}\right](\alpha)} \\
& = \begin{cases}{\left[0, q_{i}-\frac{q_{i}}{q} L^{-1}(\alpha)\right],} & \alpha \in(0, L(q)], \\
{[0,0],} & \alpha \in(L(q), 1] .\end{cases}
\end{aligned}
$$

(a) For $\alpha \in[0, L(q)]$, the $\alpha$ cut set of the retailer $i$ 's fuzzy profit is

$$
\begin{aligned}
& {\left[\widetilde{\Pi}_{R_{i}}\left(q_{i}, q_{-i}\right)\right](\alpha)} \\
& \quad=\left[p \frac{q_{i}}{q} L^{-1}(\alpha)-w q_{i}, p q_{i}+b q_{i}-b \frac{q_{i}}{q} L^{-1}(\alpha)-w q_{i}\right] .
\end{aligned}
$$

(b) For $\alpha \in(L(q), 1]$, the result turns to

$$
\left[\widetilde{\Pi}_{R_{i}}\left(q_{i}, q_{-i}\right)\right](\alpha)=\left[p q_{i}-w q_{i}, p q_{i}-w q_{i}\right] .
$$

By (7), we can get the fuzzy expected profit $E\left[\widetilde{\Pi}_{R_{i}}\left(q_{i}, q_{-i}\right)\right]$ as

$$
\begin{aligned}
& E\left[\widetilde{\Pi}_{R_{i}}\left(q_{i}, q_{-i}\right)\right] \\
& =\frac{1}{2} \int_{0}^{L(q)}\left(p \frac{q_{i}}{q} L^{-1}(\alpha)-w q_{i}+p q_{i}+b q_{i}\right. \\
& \left.\quad-b \frac{q_{i}}{q} L^{-1}(\alpha)-w q_{i}\right) \mathrm{d} \alpha \\
& \quad+\frac{1}{2} \int_{L(q)}^{1}\left(p q_{i}-w q_{i}+p q_{i}-w q_{i}\right) \mathrm{d} \alpha \\
& =\frac{1}{2}(p-b)\left(\frac{q_{i}}{q} \int_{0}^{L(q)} L^{-1}(\alpha) \mathrm{d} \alpha-q_{i} L(q)\right) \\
& \quad+(p-w) q_{i} .
\end{aligned}
$$

The first and second derivatives of $E\left[\widetilde{\Pi}_{R_{i}}\left(q_{i}, q_{-i}\right)\right]$ in (33) can be obtained as follows:

$$
\begin{aligned}
& \frac{\mathrm{d} E\left[\widetilde{\Pi}_{R_{i}}\left(q_{i}, q_{-i}\right)\right]}{\mathrm{d} q_{i}} \\
& \quad=\frac{1}{2}(p-b)\left(\frac{q-q_{i}}{q^{2}} \int_{0}^{L(q)} L^{-1}(\alpha) \mathrm{d} \alpha-L(q)\right)+p-w,
\end{aligned}
$$

$$
\begin{aligned}
& \frac{\mathrm{d}^{2} E\left[\widetilde{\Pi}_{R_{i}}\left(q_{i}, q_{-i}\right)\right]}{d_{q_{i}}^{2}} \\
& =-\frac{1}{2}(p-b)\left(\frac{q-q_{i}}{q^{3}} \int_{0}^{L(q)} L^{-1}(\alpha) \mathrm{d} \alpha+\frac{q_{i}}{q} L^{\prime}(q)\right) .
\end{aligned}
$$

Since $L(q)$ is an increasing function with $L^{\prime}(q)>0$ and $p>b$, therefore $\mathrm{d}^{2} E\left[\widetilde{\Pi}_{R_{i}}\left(q_{i}, q_{-i}\right)\right] / \mathrm{d} q_{i}^{2}$ is negative and $E\left[\widetilde{\Pi}_{R_{i}}\left(q_{i}, q_{-i}\right)\right]$ is concave in $q_{i}$. Hence, there exists an optimal order quantity for retailer $i$ for each $q_{-i}$, where $q_{-i}=\sum_{j=1, j \neq i}^{n} q_{j}$. A set of order quantity $q^{*}=\left(q_{1}^{*}, \ldots, q_{n}^{*}\right)$ is a Nash equilibrium of the decentralized system if each retailer's order quantity is a best response. Thus, any Nash equilibrium must satisfy each retailer's first-order condition. Let $\mathrm{d} E\left[\widetilde{\Pi}_{R_{i}}\left(q_{i}, q_{-i}\right)\right] / \mathrm{d} q_{i}=0$; we can get

$$
\begin{aligned}
q_{i}^{*}=q^{*} & \frac{2(p-w)}{p-b} \\
& \left.+\frac{1}{q^{*}} \int_{0}^{L\left(q^{*}\right)} L^{-1}(\alpha) \mathrm{d} \alpha-L\left(q^{*}\right)\right) \\
& \times\left(\frac{1}{q^{*}} \int_{0}^{L\left(q^{*}\right)} L^{-1}(\alpha) \mathrm{d} \alpha\right)^{-1} .
\end{aligned}
$$

Equation (35) gives each retailer's equilibrium order conditional on $q^{*}$ being the equilibrium total order quantity. Hence, (35) describes an equilibrium only if $q^{*}=n q_{i}^{*}$.

Substitute (35) into $q^{*}=n q_{i}^{*}$ and simplify

$$
L\left(q^{*}\right)-\left(\frac{n-1}{n}\right)\left(\frac{1}{q^{*}} \int_{0}^{L\left(q^{*}\right)} L^{-1}(\alpha) \mathrm{d} \alpha\right)=\frac{2(p-w)}{p-b} .
$$

In order to fully coordinate the supply chain, let $L\left(q^{*}\right)=2(p-$ $c) / p$; we can obtain

$$
\begin{aligned}
& w^{*}=p-\frac{1}{2}(p-b) \\
& \times\left(L\left(q^{*}\right)-\left(\frac{n-1}{n}\right)\left(\frac{1}{q^{*}} \int_{0}^{L\left(q^{*}\right)} L^{-1}(\alpha) \mathrm{d} \alpha\right)\right) \\
&=p-\frac{1}{2}(p-b)\left(\frac{2(p-c)}{p}-\left(\frac{n-1}{n}\right)\right. \\
&\left.\times\left(\frac{\int_{0}^{2(p-c) / p} L^{-1}(\alpha) \mathrm{d} \alpha}{L^{-1}(2(p-c) / p)}\right)\right) .
\end{aligned}
$$

The poof of Theorem 11 is completed. 
Theorem 12. When $p>2 c$, the optimal wholesale price $w^{*}$ in return contract is

$$
\begin{aligned}
w^{*}=b & +\frac{1}{2}(p-b) \\
\times & \frac{2 c}{p}+\left(\frac{n-1}{n}\right) \\
& \left.\times\left(\frac{\int_{2 c / p}^{1} R^{-1}(\alpha) \mathrm{d} \alpha+\int_{0}^{1} L^{-1}(\alpha) \mathrm{d} \alpha}{R^{-1}(2 c / p)}\right)\right) .
\end{aligned}
$$

Proof. If $q_{i} \in\left(\left(q_{i} / q\right) d_{2},\left(q_{i} / q\right) d_{3}\right]$, that is, $q \in\left(d_{2}, d_{3}\right]$, then the $\alpha$ cut sets of $\min \left\{q_{i},\left(q_{i} / q\right) \widetilde{D}\right\}$ and $\max \left\{q_{i}-\left(q_{i} / q\right) \widetilde{D}, 0\right\}$ are

$$
\begin{aligned}
& {\left[\min \left\{q_{i}, \frac{q_{i}}{q} \widetilde{D}\right\}\right](\alpha)} \\
& = \begin{cases}{\left[\frac{q_{i}}{q} L^{-1}(\alpha), q_{i}\right],} & \alpha \in[0, R(q)], \\
{\left[\frac{q_{i}}{q} L^{-1}(\alpha), \frac{q_{i}}{q} R^{-1}(\alpha)\right],} & \alpha \in(R(q), 1]\end{cases} \\
& {\left[\max \left\{q_{i}-\frac{q_{i}}{q} \widetilde{D}, 0\right\}\right](\alpha)} \\
& = \begin{cases}{\left[0, q_{i}-\frac{q_{i}}{q} L^{-1}(\alpha)\right],} & \alpha \in[0, R(q)], \\
{\left[q_{i}-\frac{q_{i}}{q} R^{-1}(\alpha), q_{i}-\frac{q_{i}}{q} L^{-1}(\alpha)\right],} & \alpha \in(R(q), 1] .\end{cases}
\end{aligned}
$$

(a) For $\alpha \in[0, R(q)]$, the $\alpha$ cut set of the retailer $i$ 's fuzzy profit is

$$
\begin{aligned}
& {\left[\widetilde{\Pi}_{R_{i}}\left(q_{i}, q_{-i}\right)\right](\alpha)} \\
& \quad=\left[p \frac{q_{i}}{q} L^{-1}(\alpha)-w q_{i}, p q_{i}+b q_{i}-b \frac{q_{i}}{q} L^{-1}(\alpha)-w q_{i}\right] .
\end{aligned}
$$

(b) For $\alpha \in(R(q), 1]$, the result turns to

$$
\begin{gathered}
{\left[\widetilde{\Pi}_{R_{i}}\left(q_{i}, q_{-i}\right)\right](\alpha)=\left[p \frac{q_{i}}{q} L^{-1}(\alpha)+b q_{i}-b \frac{q_{i}}{q} R^{-1}(\alpha)\right.} \\
-w q_{i}, p \frac{q_{i}}{q} R^{-1}(\alpha)+b q_{i} \\
\left.-b \frac{q_{i}}{q} L^{-1}(\alpha)-w q_{i}\right]
\end{gathered}
$$

By (7), we can get the fuzzy expected profit $E\left[\widetilde{\Pi}_{R_{i}}\left(q_{i}, q_{-i}\right)\right]$ as

$$
\begin{aligned}
& E\left[\widetilde{\Pi}_{R_{i}}\left(q_{i}, q_{-i}\right)\right] \\
& =\frac{1}{2} \int_{0}^{R(q)}\left(p \frac{q_{i}}{q} L^{-1}(\alpha)-w q_{i}+p q_{i}+b q_{i}\right. \\
& \left.-b \frac{q_{i}}{q} L^{-1}(\alpha)-w q_{i}\right) \mathrm{d} \alpha \\
& +\frac{1}{2} \int_{R(q)}^{1}\left(p \frac{q_{i}}{q} L^{-1}(\alpha)+b q_{i}-b \frac{q_{i}}{q} R^{-1}(\alpha)-w q_{i}\right. \\
& \left.+\frac{q_{i}}{q} R^{-1}(\alpha)+b q_{i}-b \frac{q_{i}}{q} L^{-1}(\alpha)-w q_{i}\right) \mathrm{d} \alpha \\
& =\frac{1}{2}(p-b)\left(\frac{q_{i}}{q} \int_{R(q)}^{1} R^{-1}(\alpha) \mathrm{d} \alpha\right. \\
& \left.+\frac{q_{i}}{q} \int_{0}^{1} L^{-1}(\alpha) \mathrm{d} \alpha+q_{i} R(q)\right)-(w-b) q_{i} .
\end{aligned}
$$

The first and second derivatives of $E\left[\widetilde{\Pi}_{R_{i}}\left(q_{i}, q_{-i}\right)\right]$ in $(42)$ can be obtained as follows:

$$
\begin{aligned}
& \frac{\mathrm{d} E\left[\widetilde{\Pi}_{R_{i}}\left(q_{i}, q_{-i}\right)\right]}{\mathrm{d} q_{i}} \\
& \quad=\frac{1}{2}(p-b)\left(\frac { q - q _ { i } } { q ^ { 2 } } \left(\int_{R(q)}^{1} R^{-1}(\alpha) \mathrm{d} \alpha\right.\right.
\end{aligned}
$$

$$
\left.\left.-\int_{0}^{1} L^{-1}(\alpha) \mathrm{d} \alpha\right)+R(q)\right)
$$

$$
\begin{aligned}
& \quad-(w-b), \\
& \frac{\mathrm{d}^{2} E\left[\widetilde{\Pi}_{R_{i}}\left(q_{i}, q_{-i}\right)\right]}{\mathrm{d} q_{i}{ }^{2}} \\
& =-\frac{1}{2}(p-b)\left(\frac { q _ { i } } { q ^ { 3 } } \left(\int_{R(q)}^{1} R^{-1}(\alpha) \mathrm{d} \alpha\right.\right.
\end{aligned}
$$

$$
\left.\left.-\int_{0}^{1} L^{-1}(\alpha) \mathrm{d} \alpha\right)-\frac{q_{i}}{q} R^{\prime}(q)\right) .
$$

Since $R(q)$ is a decreasing function with $R^{\prime}(q)<0$ and $p>b$, therefore $\mathrm{d}^{2} E\left[\widetilde{\Pi}_{R_{i}}\left(q_{i}, q_{-i}\right)\right] / \mathrm{d} q_{i}^{2}$ is negative and $E\left[\widetilde{\Pi}_{R_{i}}\left(q_{i}, q_{-i}\right)\right]$ is concave in $q_{i}$. 
Any Nash equilibrium must satisfy each retailer's firstorder condition. Let $\mathrm{d} E\left[\widetilde{\Pi}_{R_{i}}\left(q_{i}, q_{-i}\right)\right] / \mathrm{d} q_{i}=0$; we can get

$$
\begin{aligned}
q_{i}^{*}=q^{*} & \left(\frac{1}{q^{*}} \int_{R\left(q^{*}\right)}^{1} R^{-1}(\alpha) \mathrm{d} \alpha+\frac{1}{q^{*}} \int_{0}^{1} L^{-1}(\alpha) \mathrm{d} \alpha\right. \\
& \left.+R\left(q^{*}\right)-\frac{2(w-b)}{p-b}\right) \\
& \times\left(\frac{1}{q^{*}} \int_{R\left(q^{*}\right)}^{1} R^{-1}(\alpha) \mathrm{d} \alpha+\frac{1}{q^{*}} \int_{0}^{1} L^{-1}(\alpha) \mathrm{d} \alpha\right)^{-1} .
\end{aligned}
$$

Substitute (44) into $q^{*}=n q_{i}^{*}$ and simplify

$$
\begin{aligned}
& R\left(q^{*}\right)+\left(\frac{n-1}{n}\right) \\
& \quad \times\left(\frac{1}{q^{*}} \int_{R\left(q^{*}\right)}^{1} R^{-1}(\alpha) \mathrm{d} \alpha+\frac{1}{q^{*}} \int_{0}^{1} L^{-1}(\alpha) \mathrm{d} \alpha\right) \\
& \quad=\frac{2(w-b)}{p-b} .
\end{aligned}
$$

In order to fully coordinate the supply chain, let $R\left(q^{*}\right)=$ $2 c / p$; we can obtain

$$
\begin{aligned}
w^{*}= & b+\frac{1}{2}(p-b) \\
\times & R\left(q^{*}\right)+\left(\frac{n-1}{n}\right) \\
& \left.\times\left(\frac{1}{q^{*}} \int_{R\left(q^{*}\right)}^{1} R^{-1}(\alpha) \mathrm{d} \alpha+\frac{1}{q^{*}} \int_{0}^{1} L^{-1}(\alpha) \mathrm{d} \alpha\right)\right) \\
= & \frac{1}{2}(p-b) \\
& \times\left(\begin{array}{c}
\frac{2 c}{p}+\left(\frac{n-1}{n}\right) \\
\times\left(\frac{\int_{2 c / p}^{1} R^{-1}(\alpha) \mathrm{d} \alpha+\int_{0}^{1} L^{-1}(\alpha) \mathrm{d} \alpha}{R^{-1}(2 c / p)}\right)
\end{array}\right) .
\end{aligned}
$$

The poof of Theorem 12 is completed.
Theorem 13. In fuzzy return contract, the retailer $i(i=$ $1,2, \ldots, n)$ and the supplier attain their optimal fuzzy expected value of the profits at $w^{*}$ in fuzzy return contract, where

$$
\begin{aligned}
E\left[\widetilde{\Pi}_{R_{i}}\left(q_{i}, q_{-i}\right)\right]^{*} & =\left(\frac{p-b}{p n^{2}}\right) E\left[\widetilde{\Pi}_{S C}\right]^{*}, \quad i=1,2, \ldots, n, \\
E\left[\widetilde{\Pi}_{S}\right]^{*} & =\left(\frac{p(n-1)+b}{p n}\right) E\left[\widetilde{\Pi}_{S C}\right]^{*} .
\end{aligned}
$$

Proof. Consider the following.

Case $1\left(d_{1} \leq q \leq d_{2}\right)$. When $d_{1} \leq q \leq d_{2}$, substituting $w^{*}$ and $L\left(q^{*}\right)=2(p-c) / p$ into (33), the fuzzy expected profit of the retailer $i(i=1,2, \ldots, n)$ is given as

$$
\begin{aligned}
E\left[\widetilde{\Pi}_{R_{i}}\left(q_{i}, q_{-i}\right)\right]^{*} & =\frac{p-b}{2 n^{2}} \int_{0}^{2(p-c) / p} L^{-1}(\alpha) \mathrm{d} \alpha \\
& =\left(\frac{p-b}{p n^{2}}\right) E\left[\widetilde{\Pi}_{\mathrm{SC}}\right]^{*} .
\end{aligned}
$$

The fuzzy expected profit of the supplier is

$$
\begin{aligned}
E\left[\widetilde{\Pi}_{S}\right]^{*} & =E\left[\widetilde{\Pi}_{\mathrm{SC}}\right]^{*}-n E\left[\widetilde{\Pi}_{R_{i}}\left(q_{i}, q_{-i}\right)\right]^{*} \\
& =\left(\frac{p(n-1)+b}{p n}\right) E\left[\widetilde{\Pi}_{\mathrm{SC}}\right]^{*} .
\end{aligned}
$$

Case $2\left(d_{2} \leq q \leq d_{3}\right)$. When $d_{2}<q \leq d_{3}$, substituting $w^{*}$ and $R\left(q^{*}\right)=2 c / p$ into (42), the fuzzy expected profit of the retailer $i(i=1,2, \ldots, n)$ is given as

$$
\begin{aligned}
E & {\left[\widetilde{\Pi}_{R_{i}}\left(q_{i}, q_{-i}\right)\right]^{*} } \\
& =\frac{p-b}{2 n^{2}}\left(\int_{2 c / p}^{1} R^{-1}(\alpha) \mathrm{d} \alpha+\int_{0}^{1} L^{-1}(\alpha) \mathrm{d} \alpha\right) \\
& =\left(\frac{p-b}{p n^{2}}\right) E\left[\widetilde{\Pi}_{\mathrm{SC}}\right]^{*} .
\end{aligned}
$$

The fuzzy expected profit of the supplier is

$$
\begin{aligned}
E\left[\widetilde{\Pi}_{S}\right]^{*} & =E\left[\widetilde{\Pi}_{\mathrm{SC}}\right]^{*}-n E\left[\widetilde{\Pi}_{R_{i}}\left(q_{i}, q_{-i}\right)\right]^{*} \\
& =\left(\frac{p(n-1)+b}{p n}\right) E\left[\widetilde{\Pi}_{\mathrm{SC}}\right]^{*} .
\end{aligned}
$$

The poof of Theorem 13 is completed.

3.3. Revenue-Sharing Contract with Fuzzy Demand. In a revenue-sharing contract, the retailer $i(i=1,2, \ldots, n)$ shares with the supplier a percentage of his revenue. Let $(1-\Phi)$ be the fraction the supplier earns, and then $\Phi$ is the fraction the retailer $i$ keeps. 
Thus, we can express the fuzzy profit of the retailer $i$ as follows:

$$
\begin{aligned}
\widetilde{\Pi}_{R_{i}}\left(q_{i}, q_{-i}\right) & =p \Phi \min \left\{q_{i}, \widetilde{D}_{i}\right\}-w q_{i} \\
& =p \Phi_{i} \min \left\{q_{i}, \frac{q_{i}}{q} \widetilde{D}\right\}-w q_{i}
\end{aligned}
$$

where $q=\sum_{i=1}^{n} q_{i}$ and $q_{-i}=q-q_{i}$.

The retailer $i(i=1,2, \ldots, n)$ tries to maximize its fuzzy expected profit $E\left[\widetilde{\Pi}_{R_{i}}\left(q_{i}, q_{-i}\right)\right]$ in revenue-sharing contract by choosing the optimal order quantity $q_{i}$, which solves the following model:

$$
\begin{array}{r}
E\left[\widetilde{\Pi}_{R_{i}}\left(q_{i}, q_{-i}\right)\right]=E\left[p \Phi \min \left\{q_{i}, \frac{q_{i}}{q} \widetilde{D}\right\}-w q_{i}\right] \\
\text { s.t. } \frac{q_{i}}{q} d_{1} \leq q_{i} \leq \frac{q_{i}}{q} d_{3} .
\end{array}
$$

Theorem 14. When $c<p \leq 2 c$, the optimal wholesale price $w^{* *}$ in revenue-sharing contract is

$$
\begin{aligned}
w^{* *}= & p \Phi-\frac{1}{2} p \Phi \\
& \times\left(\frac{2(p-c)}{p}-\left(\frac{n-1}{n}\right)\right. \\
& \left.\times\left(\frac{\int_{0}^{2(p-c) / p} L^{-1}(\alpha) \mathrm{d} \alpha}{L^{-1}(2(p-c) / p)}\right)\right) .
\end{aligned}
$$

Proof. If $q_{i} \in\left[\left(q_{i} / q\right) d_{1},\left(q_{i} / q\right) d_{2}\right]$, that is, $q \in\left[d_{1}, d_{2}\right]$, then the $\alpha$ cut set of $\min \left\{q_{i},\left(q_{i} / q\right) \widetilde{D}\right\}$ is

$$
\begin{aligned}
& {\left[\min \left\{q_{i}, \frac{q_{i}}{q} \widetilde{D}\right\}\right](\alpha)} \\
& \quad= \begin{cases}{\left[\frac{q_{i}}{q} L^{-1}(\alpha), q_{i}\right],} & \alpha \in(0, L(q)], \\
{\left[q_{i}, q_{i}\right],} & \alpha \in(L(q), 1] .\end{cases}
\end{aligned}
$$

(a) For $\alpha \in[0, L(q)]$, the $\alpha$ cut set of the retailer $i$ 's fuzzy profit is

$$
\left[\widetilde{\Pi}_{R_{i}}\left(q_{i}, q_{-i}\right)\right](\alpha)=\left[p \Phi \frac{q_{i}}{q} L^{-1}(\alpha)-w q_{i}, p \Phi q_{i}-w q_{i}\right]
$$

(b) For $\alpha \in(L(q), 1]$, the result turns to

$$
\left[\widetilde{\Pi}_{R_{i}}\left(q_{i}, q_{-i}\right)\right](\alpha)=\left[p \Phi q_{i}-w q_{i}, p \Phi q_{i}-w q_{i}\right]
$$

By (7), we can get the fuzzy expected profit $E\left[\widetilde{\Pi}_{R_{i}}\left(q_{i}, q_{-i}\right)\right]$ as

$$
\begin{aligned}
E\left[\widetilde{\Pi}_{R_{i}}\left(q_{i}, q_{-i}\right)\right] \\
=\frac{1}{2} \int_{0}^{L(q)}\left(p \Phi \frac{q_{i}}{q} L^{-1}(\alpha)-w q_{i}+p \Phi q_{i}-w q_{i}\right) \mathrm{d} \alpha \\
\quad+\frac{1}{2} \int_{L(q)}^{1}\left(p \Phi q_{i}-w q_{i}+p \Phi q_{i}-w q_{i}\right) \mathrm{d} \alpha \\
=\frac{1}{2} p \Phi\left(\frac{q_{i}}{q} \int_{0}^{L(q)} L^{-1}(\alpha) \mathrm{d} \alpha-q_{i} L(q)\right)+(p \Phi-w) q_{i} .
\end{aligned}
$$

The first and second derivatives of $E\left[\widetilde{\Pi}_{R_{i}}\left(q_{i}, q_{-i}\right)\right]$ in (58) can be obtained as follows:

$$
\begin{aligned}
& \frac{\mathrm{d} E\left[\widetilde{\Pi}_{R_{i}}\left(q_{i}, q_{-i}\right)\right]}{\mathrm{d} q_{i}} \\
& =\frac{1}{2} p \Phi\left(\frac{q-q_{i}}{q^{2}} \int_{0}^{L(q)} L^{-1}(\alpha) \mathrm{d} \alpha-L(q)\right)+p \Phi-w, \\
& \frac{\mathrm{d}^{2} E\left[\widetilde{\Pi}_{R_{i}}\left(q_{i}, q_{-i}\right)\right]}{\mathrm{d} q_{i}^{2}} \\
& =-\frac{1}{2} p \Phi\left(\frac{q-q_{i}}{q^{3}} \int_{0}^{L(q)} L^{-1}(\alpha) \mathrm{d} \alpha+\frac{q_{i}}{q} L^{\prime}(q)\right) .
\end{aligned}
$$

Since $L(q)$ is an increasing function with $L^{\prime}(q)>0$ and $\Phi>0$, therefore $\mathrm{d}^{2} E\left[\widetilde{\Pi}_{R_{i}}\left(q_{i}, q_{-i}\right)\right] / \mathrm{d} q_{i}^{2}$ is negative and $E\left[\widetilde{\Pi}_{R_{i}}\left(q_{i}, q_{-i}\right)\right]$ is concave in $q_{i}$. Hence, there exists an optimal order quantity for retailer $i$ for each $q_{-i}$. A set of order quantity, $q^{*}=\left(q_{1}^{*}, \ldots, q_{n}^{*}\right)$ is a Nash equilibrium of the decentralized system if each retailer's order quantity is a best response.

Any Nash equilibrium must satisfy each retailer's firstorder condition. Let $\mathrm{d} E\left[\widetilde{\Pi}_{R_{i}}\left(q_{i}, q_{-i}\right)\right] / \mathrm{d} q_{i}=0$; we can get

$$
q_{1}^{q_{1}^{* *}} \frac{2(p \Phi-w) / p \Phi+\left(1 / q^{*}\right) \int_{0}^{L\left(q^{*}\right)} L^{-1}(\alpha) \mathrm{d} \alpha-L\left(q^{*}\right)}{\left(1 / q^{*}\right) \int_{0}^{L\left(q^{*}\right)} L^{-1}(\alpha) \mathrm{d} \alpha} .
$$

Equation (60) gives each retailer's equilibrium order conditional on $q^{*}$ being the equilibrium total order quantity. Hence, (60) describes an equilibrium only if $q^{*}=n q_{i}^{* *}$.

Substitute (60) into $q^{*}=n q_{i}^{* *}$ and simplify

$$
L\left(q^{*}\right)-\left(\frac{n-1}{n}\right)\left(\frac{1}{q^{*}} \int_{0}^{L\left(q^{*}\right)} L^{-1}(\alpha) \mathrm{d} \alpha\right)=\frac{2(p \Phi-w)}{p \Phi}
$$


In order to fully coordinate the supply chain, let $L\left(q^{*}\right)=2(p-$ $c) / p$; we can obtain

$$
\begin{aligned}
w^{* *}= & p \Phi-\frac{1}{2} p \Phi \\
& \times\left(L\left(q^{*}\right)-\left(\frac{n-1}{n}\right)\left(\frac{1}{q^{*}} \int_{0}^{L\left(q^{*}\right)} L^{-1}(\alpha) \mathrm{d} \alpha\right)\right) \\
= & p \Phi-\frac{1}{2} p \Phi \\
& \times\left(\begin{array}{c}
\frac{2(p-c)}{p}-\left(\frac{n-1}{n}\right) \\
\quad \\
\quad\left(\frac{\int_{0}^{2(p-c) / p} L^{-1}(\alpha) \mathrm{d} \alpha}{L^{-1}(2(p-c) / p)}\right)
\end{array}\right) .
\end{aligned}
$$

The poof of Theorem 14 is completed.

Theorem 15. When $p>2 c$, the optimal wholesale price $w^{* *}$ in revenue-sharing contract is

$$
\begin{aligned}
w^{* *}= & \frac{1}{2} p \Phi \\
& \times\left(\frac{2 c}{p}+\left(\frac{n-1}{n}\right)\right. \\
& \left.\quad \times\left(\frac{\int_{2 c / p}^{1} R^{-1}(\alpha) \mathrm{d} \alpha+\int_{0}^{1} L^{-1}(\alpha) \mathrm{d} \alpha}{R^{-1}(2 c / p)}\right)\right) .
\end{aligned}
$$

Proof. If $q_{i} \in\left(\left(q_{i} / q\right) d_{2},\left(q_{i} / q\right) d_{3}\right]$, that is, $q \in\left(d_{2}, d_{3}\right]$, then the $\alpha$ cut set of min $\left\{q_{i},\left(q_{i} / q\right) \widetilde{D}\right\}$ is

$$
\begin{aligned}
& {\left[\min \left\{q_{i}, \frac{q_{i}}{q} \widetilde{D}\right\}\right](\alpha)} \\
& = \begin{cases}{\left[\frac{q_{i}}{q} L^{-1}(\alpha), q_{i}\right],} & \alpha \in[0, R(q)], \\
{\left[\frac{q_{i}}{q} L^{-1}(\alpha), \frac{q_{i}}{q} R^{-1}(\alpha)\right],} & \alpha \in(R(q), 1] .\end{cases}
\end{aligned}
$$

(a) For $\alpha \in[0, R(q)]$, the $\alpha$ cut set of the retailer $i$ 's fuzzy profit is

$$
\left[\widetilde{\Pi}_{R_{i}}\left(q_{i}, q_{-i}\right)\right](\alpha)=\left[p \Phi \frac{q_{i}}{q} L^{-1}(\alpha)-w q_{i}, p q_{i}-w q_{i}\right] .
$$

(b) For $\alpha \in(R(q), 1]$, the result turns to

$$
\begin{aligned}
& {\left[\widetilde{\Pi}_{R_{i}}\left(q_{i}, q_{-i}\right)\right](\alpha)} \\
& \quad=\left[p \Phi \frac{q_{i}}{q} L^{-1}(\alpha)-w q_{i}, p \Phi \frac{q_{i}}{q} R^{-1}(\alpha)-w q_{i}\right] .
\end{aligned}
$$

By (7), we can get the fuzzy expected profit $E\left[\widetilde{\Pi}_{R_{i}}\left(q_{i}, q_{-i}\right)\right]$ as

$$
\begin{aligned}
& E\left[\widetilde{\Pi}_{R_{i}}\left(q_{i}, q_{-i}\right)\right] \\
& =\frac{1}{2} \int_{0}^{R(q)}\left(p \Phi \frac{q_{i}}{q} L^{-1}(\alpha)-w q_{i}+p \Phi q_{i}-w q_{i}\right) \mathrm{d} \alpha \\
& \quad+\frac{1}{2} \int_{R(q)}^{1}\left(p \Phi \frac{q_{i}}{q} L^{-1}(\alpha)-w q_{i}+p \Phi \frac{q_{i}}{q} R^{-1}(\alpha)-w q_{i}\right) \mathrm{d} \alpha \\
& =\frac{1}{2} p \Phi\left(\frac{q_{i}}{q} \int_{R(q)}^{1} R^{-1}(\alpha) \mathrm{d} \alpha\right. \\
& \left.\quad+\frac{q_{i}}{q} \int_{0}^{1} L^{-1}(\alpha) \mathrm{d} \alpha+q_{i} R(q)\right)-w q_{i} .
\end{aligned}
$$

The first and second derivatives of $E\left[\widetilde{\Pi}_{R_{i}}\left(q_{i}, q_{-i}\right)\right]$ in $(67)$ can be obtained as follows:

$$
\begin{aligned}
& \frac{\mathrm{d} E\left[\widetilde{\Pi}_{R_{i}}\left(q_{i}, q_{-i}\right)\right]}{\mathrm{d} q_{i}} \\
& =\frac{1}{2} p \Phi\left(\frac { q - q _ { i } } { q ^ { 2 } } \left(\int_{R(q)}^{1} R^{-1}(\alpha) \mathrm{d} \alpha\right.\right.
\end{aligned}
$$

$$
\left.\left.-\int_{0}^{1} L^{-1}(\alpha) \mathrm{d} \alpha\right)+R(q)\right)-w
$$

$$
\begin{aligned}
& \frac{\mathrm{d}^{2} E\left[\widetilde{\Pi}_{R_{i}}\left(q_{i}, q_{-i}\right)\right]}{\mathrm{d} q_{i}^{2}} \\
&=-\frac{1}{2} p \Phi\left(\frac { q _ { i } } { q ^ { 3 } } \left(\int_{R(q)}^{1} R^{-1}(\alpha) \mathrm{d} \alpha\right.\right. \\
&\left.\left.\quad-\int_{0}^{1} L^{-1}(\alpha) \mathrm{d} \alpha\right)-\frac{q_{i}}{q} R^{\prime}(q)\right) .
\end{aligned}
$$

Since $R(q)$ is a decreasing function with $R^{\prime}(q)<0$ and $\Phi>0$, therefore $\mathrm{d}^{2} E\left[\widetilde{\Pi}_{R_{i}}\left(q_{i}, q_{-i}\right)\right] / \mathrm{d} q_{i}^{2}$ is negative and $E\left[\widetilde{\Pi}_{R_{i}}\left(q_{i}, q_{-i}\right)\right]$ is concave in $q_{i}$. 
Any Nash equilibrium must satisfy each retailer's firstorder condition. Let $\mathrm{d} E\left[\widetilde{\Pi}_{R_{i}}\left(q_{i}, q_{-i}\right)\right] / \mathrm{d} q_{i}=0$; we can get

$$
\begin{aligned}
q_{i}^{* *}= & q^{*}\left(\frac{1}{q^{*}} \int_{R\left(q^{*}\right)}^{1} R^{-1}(\alpha) \mathrm{d} \alpha\right. \\
& \left.\quad+\frac{1}{q^{*}} \int_{0}^{1} L^{-1}(\alpha) \mathrm{d} \alpha+R\left(q^{*}\right)-\frac{2 w}{p \Phi}\right) \\
& \times\left(\frac{1}{q^{*}} \int_{R\left(q^{*}\right)}^{1} R^{-1}(\alpha) \mathrm{d} \alpha+\frac{1}{q^{*}} \int_{0}^{1} L^{-1}(\alpha) \mathrm{d} \alpha\right)^{-1} .
\end{aligned}
$$

Substitute (69) into $q^{*}=n q_{i}^{* *}$ and simplify

$$
\begin{aligned}
& R\left(q^{*}\right)+\left(\frac{n-1}{n}\right) \\
& \quad \times\left(\frac{1}{q^{*}} \int_{R\left(q^{*}\right)}^{1} R^{-1}(\alpha) \mathrm{d} \alpha+\frac{1}{q^{*}} \int_{0}^{1} L^{-1}(\alpha) \mathrm{d} \alpha\right)=\frac{2 w}{p \Phi}
\end{aligned}
$$

In order to fully coordinate the supply chain, let $R\left(q^{*}\right)=$ $2 c / p$; we can obtain

$$
\begin{aligned}
w^{* *}=\frac{1}{2} p \Phi( & R\left(q^{*}\right)+\left(\frac{n-1}{n}\right) \\
& \times\left(\frac{1}{q^{*}} \int_{R\left(q^{*}\right)}^{1} R^{-1}(\alpha) \mathrm{d} \alpha\right. \\
& \left.\left.+\frac{1}{q^{*}} \int_{0}^{1} L^{-1}(\alpha) \mathrm{d} \alpha\right)\right) \\
=\frac{1}{2} p \Phi\left(\frac{2 c}{p}+\left(\frac{n-1}{n}\right)\right. & \left.\left(\frac{\int_{2 c / p}^{1} R^{-1}(\alpha) \mathrm{d} \alpha+\int_{0}^{1} L^{-1}(\alpha) \mathrm{d} \alpha}{R^{-1}(2 c / p)}\right)\right)
\end{aligned}
$$

The poof of Theorem 15 is completed.

Theorem 16. In fuzzy revenue-sharing contract, the retailer $i(i=1,2, \ldots, n)$ and the supplier attain their optimal fuzzy expected value of the profits at $w^{* *}$ in fuzzy return contract, where

$$
\begin{gathered}
E\left[\widetilde{\Pi}_{R_{i}}\left(q_{i}, q_{-i}\right)\right]^{* *}=\left(\frac{\Phi}{n^{2}}\right) E\left[\widetilde{\Pi}_{S C}\right]^{*}, \quad i=1,2, \ldots, n, \\
E\left[\widetilde{\Pi}_{S}\right]^{* *}=\left(\frac{n-\Phi}{n}\right) E\left[\widetilde{\Pi}_{S C}\right]^{*} .
\end{gathered}
$$

Proof. Consider the following.

Case $1\left(d_{1} \leq q \leq d_{2}\right)$. When $d_{1} \leq q \leq d_{2}$, substituting $w^{* *}$ and $L\left(q^{*}\right)=2(p-c) / p$ into (35), the fuzzy expected profit of the retailer $i(i=1,2, \ldots, n)$ is given as

$$
\begin{aligned}
E\left[\widetilde{\Pi}_{R_{i}}\left(q_{i}, q_{-i}\right)\right]^{* *} & =\frac{p \Phi}{2 n^{2}} \int_{0}^{2(p-c) / p} L^{-1}(\alpha) \mathrm{d} \alpha \\
& =\left(\frac{\Phi}{n^{2}}\right) E\left[\widetilde{\Pi}_{\mathrm{SC}}\right]^{*} .
\end{aligned}
$$

The fuzzy expected profit of the supplier is

$$
\begin{aligned}
E\left[\widetilde{\Pi}_{S}\right]^{* *} & =E\left[\widetilde{\Pi}_{\mathrm{SC}}\right]^{*}-n E\left[\widetilde{\Pi}_{R_{i}}\left(q_{i}, q_{-i}\right)\right]^{* *} \\
& =\left(\frac{n-\Phi}{n}\right) E\left[\widetilde{\Pi}_{\mathrm{SC}}\right]^{*} .
\end{aligned}
$$

Case $2\left(d_{2}<q \leq d_{3}\right)$. When $d_{2}<q \leq d_{3}$, substituting $w^{* *}$ and $R\left(q^{*}\right)=2 c / p$ into (44), the fuzzy expected profit of the retailer $i(i=1,2, \ldots, n)$ is given as

$$
\begin{aligned}
E & {\left[\widetilde{\Pi}_{R_{i}}\left(q_{i}, q_{-i}\right)\right]^{* *} } \\
& =\frac{p \Phi}{2 n^{2}}\left(\int_{2 c / p}^{1} R^{-1}(\alpha) \mathrm{d} \alpha+\int_{0}^{1} L^{-1}(\alpha) \mathrm{d} \alpha\right) \\
& =\left(\frac{\Phi}{n^{2}}\right) E\left[\widetilde{\Pi}_{\mathrm{SC}}\right]^{*} .
\end{aligned}
$$

The fuzzy expected profit of the supplier is

$$
\begin{aligned}
E\left[\widetilde{\Pi}_{S}\right]^{* *} & =E\left[\widetilde{\Pi}_{\mathrm{SC}}\right]^{*}-n E\left[\widetilde{\Pi}_{R_{i}}\left(q_{i}, q_{-i}\right)\right]^{* *} \\
& =\left(\frac{n-\Phi}{n}\right) E\left[\widetilde{\Pi}_{\mathrm{SC}}\right]^{*} .
\end{aligned}
$$

The poof of Theorem 16 is completed.

Theorem 17. If $b=p(1-\Phi)$, then the fuzzy expected profits for retailer $i(i=1,2, \ldots, n)$ and supplier in return contract are equal to those in revenue-sharing contract:

$$
\begin{aligned}
E\left[\widetilde{\Pi}_{R_{i}}\left(q_{i}, q_{-i}\right)\right]^{* *} & =E\left[\widetilde{\Pi}_{R_{i}}\left(q_{i}, q_{-i}\right)\right]^{*}, \\
E\left[\widetilde{\Pi}_{S}\right]^{* *} & =E\left[\widetilde{\Pi}_{S}\right]^{*} .
\end{aligned}
$$

Proof. Substituting $b=p(1-\Phi)$ into $E\left[\widetilde{\Pi}_{R_{i}}\left(q_{i}, q_{-i}\right)\right]^{*}=((p-$ $\left.b) / p n^{2}\right) E\left[\widetilde{\Pi}_{\mathrm{SC}}\right]^{*}$ and $E\left[\widetilde{\Pi}_{S}\right]^{*}=((p(n-1)+b) /(p n)) E\left[\widetilde{\Pi}_{\mathrm{SC}}\right]^{*}$, we can obtain

$$
\begin{gathered}
E\left[\widetilde{\Pi}_{R_{i}}\left(q_{i}, q_{-i}\right)\right]^{*}=\left(\frac{\Phi}{n^{2}}\right) E\left[\widetilde{\Pi}_{\mathrm{SC}}\right]^{*}=E\left[\widetilde{\Pi}_{R_{i}}\left(q_{i}, q_{-i}\right)\right]^{* *}, \\
E\left[\widetilde{\Pi}_{S}\right]^{*}=\left(\frac{n-\Phi}{n}\right) E\left[\widetilde{\Pi}_{\mathrm{SC}}\right]^{*}=E\left[\widetilde{\Pi}_{S}\right]^{* *} .
\end{gathered}
$$

The poof of Theorem 17 is completed. 
TABLE 1: Equilibrium value of the parameters for different $c$ and $n$ in return contract $(b=30.00)$.

\begin{tabular}{lccccc}
\hline$c$ & $n$ & $q_{i}^{*}$ & $w^{*}$ & $E\left[\widetilde{\Pi}_{R_{i}}\left(q_{i}, q_{-i}\right)\right]^{*}$ & $E\left[\widetilde{\Pi}_{S}\right]^{*}$ \\
\hline \multirow{4}{*}{40.00} & 1 & 2200.00 & 58.00 & 672000.00 & 28800.00 \\
& 2 & 1100.00 & 73.27 & 168000.00 & 62400.00 \\
& 3 & 733.33 & 78.36 & 74666.67 & 73600.00 \\
& 4 & 550.00 & 80.91 & 42000.00 & 79200.00 \\
& 5 & 440.00 & 82.47 & 26880.00 & 82560.00 \\
\hline \multirow{6}{*}{50.00} & 1 & 2000.00 & 65.00 & 52500.00 & 22500.00 \\
& 2 & 1000.00 & 78.13 & 13125.00 & 48750.00 \\
& 3 & 666.67 & 82.50 & 5833.33 & 57500.00 \\
& 4 & 500.00 & 84.69 & 3289.25 & 61875.00 \\
& 5 & 400.00 & 86.00 & 2100.00 & 64500.00 \\
\hline & 1 & 1800.00 & 72.00 & 39200.00 & 16800.00 \\
60.00 & 2 & 900.00 & 82.89 & 9800.00 & 36400.00 \\
& 3 & 600.00 & 86.52 & 4355.56 & 42933.33 \\
& 4 & 450.00 & 88.33 & 2450.00 & 46200.00 \\
& 5 & 360.00 & 89.42 & 1568.00 & 48160.00 \\
\hline
\end{tabular}

\section{Numerical Example}

In this section, we tend to further elucidate the previously proposed two contracts with a numerical example. We will analyze the effect of the number of retailer $n$ on the other parameters.

Suppose the most possible value of the demand in market is $d_{2}=2000$ units and the maximum and minimum possible values of the demand are, respectively, $d_{1}=1000$ units and $d_{3}=3000$ units; that is to say, the fuzzy demand is $\widetilde{D}=$ $(1000,2000,3000)$. Let $p=100.00 \$$ per unit.

The optimal order quantity, wholesale price, and fuzzy expected profit of the actors in return contract and revenuesharing contract can be listed in Tables 1 and 2, respectively.

From Tables 1 and 2, we analyze the influence of parameters $c$ and $n$ on the optimal equilibrium values as shown below.

(a) It is obvious that the optimal order quantity for retailer $i$ will decrease along with the rise of the supplier's production cost $c$ and the number of retailers $n$ when the other parameters are fixed. Particulary, in this numerical example, the optimal total order quantity $q^{*}$ is equal to the most possible value of fuzzy demand when $c=50$. When $c>50$ and $c<50$, the optimal total order quantity of retailer is located at the left and right of the most possible value of fuzzy demand $\widetilde{D}$, respectively. The optimal wholesale price will increase along with the supplier's production $\operatorname{cost} c$ and thenumber of retailers $n$ when the other parameters are fixed.

(b) From Tables 1 and 2, it can be noted that when the other parameters are fixed in both contracts, the fuzzy expected profit of the retailer $i$ will decrease along with the rise of parameters $c$ and $n$. On the other hand, the fuzzy expected profit of the supplier will decrease along with the rise of $c$ and increase along with the rise of $n$.
TABLE 2: Equilibrium value of the parameters for different $c$ and $n$ in revenue sharing contract $(\Phi=0.60)$.

\begin{tabular}{cccccc}
\hline$c$ & $n$ & $q_{i}^{* *}$ & $w^{* *}$ & $E\left[\widetilde{\Pi}_{R_{i}}\left(q_{i}, q_{-i}\right)\right]^{* *}$ & $E\left[\widetilde{\Pi}_{S}\right]^{* *}$ \\
\hline \multirow{4}{*}{40.00} & 1 & 2200.00 & 24.00 & 57600.00 & 38400.00 \\
& 2 & 1100.00 & 37.09 & 14400.00 & 67200.00 \\
& 3 & 733.33 & 41.45 & 6400.00 & 76800.00 \\
& 4 & 550.00 & 43.64 & 3600.00 & 81600.00 \\
& 5 & 440.00 & 44.95 & 2304.00 & 84480.00 \\
\hline \multirow{6}{*}{50.00} & 1 & 2000.00 & 30.00 & 45000.00 & 30000.00 \\
& 2 & 1000.00 & 41.25 & 11250.00 & 52500.00 \\
& 3 & 666.67 & 45.00 & 5000.00 & 60000.00 \\
& 4 & 500.00 & 46.88 & 2812.50 & 63750.00 \\
& 5 & 400.00 & 48.00 & 1800.00 & 66000.00 \\
\hline \multirow{6}{*}{60.00} & 1 & 1800.00 & 36.00 & 33600.00 & 22400.00 \\
& 2 & 900.00 & 46.33 & 8400.00 & 39200.00 \\
& 3 & 600.00 & 48.44 & 3733.33 & 44800.00 \\
& 4 & 450.00 & 50.00 & 2100.00 & 47600.00 \\
& 5 & 360.00 & 50.93 & 1344.00 & 49280.00 \\
\hline
\end{tabular}

\section{Conclusions}

This paper formulates supply chain contracts based on fuzzy set theory, where a supplier and multiple competing retailers adopt return contract and revenue-sharing contract. In order to examine models performance in fuzzy demand, we use fuzzy cut sets method to solve this problem. The advantage of the proposed method is that it removes the need for enumeration over alternative values and provides a better understanding of the relations among the decision parameters. The technique proposed in this paper is easier to implement and requires less data. It is appropriate when the environment is complex, ambiguous, or there is a lack of statistical data.

\section{Acknowledgments}

The author wishes to express his sincerest thanks to the editors and anonymous referees for their constructive comments and suggestions on this paper. This work was supported by the National Natural Science Foundation of China (70972005, 71071018) and the Doctoral Foundation of Heze University (XY12BS03).

\section{References}

[1] B. A. Pasternack, "Optimal pricing and return policies for perishable commodities," Marketing Science, vol. 27, no. 1, pp. 133-140, 2008.

[2] M. K. Mantrala and K. Raman, "Demand uncertainty and supplier's returns policies for a multi-store style-good retailer," European Journal of Operational Research, vol. 115, no. 2, pp. 270-284, 1999.

[3] T. A. Taylor, "Channel coordination under price protection, midlife returns, and end-of-life returns in dynamic markets," Management Science, vol. 47, no. 9, pp. 1220-1234, 2001.

[4] H. L. Lee, V. Padmanabhan, T. A. Taylor, and S. Whang, "Price protection in the personal computer industry," Management Science, vol. 46, no. 4, pp. 467-482, 2000. 
[5] D.-Q. Yao, X. Yue, X. Wang, and J. J. Liu, "The impact of information sharing on a returns policy with the addition of a direct channel," International Journal of Production Economics, vol. 97, no. 2, pp. 196-209, 2005.

[6] X. Yue and S. Raghunathan, "The impacts of the full returns policy on a supply chain with information asymmetry," European Journal of Operational Research, vol. 180, no. 2, pp. 630647, 2007.

[7] I. Bose and P. Anand, "On returns policies with exogenous price," European Journal of Operational Research, vol. 178, no. 3, pp. 782-788, 2007.

[8] Z. Yao, Y. Wu, and K. K. Lai, "Demand uncertainty and manufacturer returns policies for style-good retailing competition," Production Planning and Control, vol. 16, no. 7, pp. 691-700, 2005.

[9] D. Ding and J. Chen, "Coordinating a three level supply chain with flexible return policies," Omega, vol. 36, no. 5, pp. 865-876, 2008.

[10] Z. Yao, S. C. H. Leung, and K. K. Lai, "Analysis of the impact of price-sensitivity factors on the returns policy in coordinating supply chain," European Journal of Operational Research, vol. 187, no. 1, pp. 275-282, 2008.

[11] D. A. Mollenkopf, E. Rabinovich, T. M. Laseter, and K. K. Boyer, "Managing internet product returns: a focus on effective service operations," Decision Sciences, vol. 38, no. 2, pp. 215-250, 2007.

[12] J. Chen and P. C. Bell, "The impact of customer returns on pricing and order decisions," European Journal of Operational Research, vol. 195, no. 1, pp. 280-295, 2009.

[13] J. Chen and P. C. Bell, "Coordinating a decentralized supply chain with customer returns and price-dependent stochastic demand using a buyback policy," European Journal of Operational Research, vol. 212, no. 2, pp. 293-300, 2011.

[14] J. Chen, "Returns with wholesale-price-discount contract in a newsvendor problem," International Journal of Production Economics, vol. 130, no. 1, pp. 104-111, 2011.

[15] X. Z. Ai, J. Chen, H. X. Zhao, and X. W. Tang, "Competition among supply chains: implication of full returns policy," International Journal of Production Economics, vol. 139, no. 1, pp. 257265, 2012.

[16] I. Giannoccaro and P. Pontrandolfo, "Supply chain coordination by revenue sharing contracts," International Journal of Production Economics, vol. 89, no. 2, pp. 131-139, 2004.

[17] G. P. Cachon and M. A. Lariviere, "Supply chain coordination with revenue-sharing contracts: strengths and limitations," Management Science, vol. 51, no. 1, pp. 30-44, 2005.

[18] D. Gupta and W. Weerawat, "Supplier-manufacturer coordination in capacitated two-stage supply chains," European Journal of Operational Research, vol. 175, no. 1, pp. 67-89, 2006.

[19] Z. Yao, S. C. H. Leung, and K. K. Lai, "Manufacturer's revenuesharing contract and retail competition," European Journal of Operational Research, vol. 186, no. 2, pp. 637-651, 2008.

[20] C. T. Linh and Y. Hong, "Channel coordination through a revenue sharing contract in a two-period newsboy problem," European Journal of Operational Research, vol. 198, no. 3, pp. 822829, 2009.

[21] B. Van Der Rhee, J. A. A. Van Der Veen, V. Venugopal, and V. R. Nalla, "A new revenue sharing mechanism for coordinating multi-echelon supply chains," Operations Research Letters, vol. 38, no. 4, pp. 296-301, 2010.

[22] F. El Ouardighi and B. Kim, "Supply quality management with wholesale price and revenue-sharing contracts under horizontal competition," European Journal of Operational Research, vol. 206, no. 2, pp. 329-340, 2010.

[23] H. Krishnan and R. A. Winter, "On the role of revenue-sharing contracts in supply chains," Operations Research Letters, vol. 39, no. 1, pp. 28-31, 2011.

[24] J.-B. Sheu, "Marketing-driven channel coordination with revenue-sharing contracts under price promotion to endcustomers," European Journal of Operational Research, vol. 214, no. 2, pp. 246-255, 2011.

[25] W.-G. Zhang, J. Fu, H. Li, and W. Xu, "Coordination of supply chain with a revenue-sharing contract under demand disruptions when retailers compete," International Journal of Production Economics, vol. 138, no. 1, pp. 68-75, 2012.

[26] O. D. Palsule-Desai, "Supply chain coordination using revenuedependent revenue sharing contracts," Omega, vol. 41, no. 4, pp. 780-796, 2013.

[27] B. Liu and Y.-K. Liu, "Expected value of fuzzy variable and fuzzy expected value models," IEEE Transactions on Fuzzy Systems, vol. 10, no. 4, pp. 445-450, 2002.

[28] Y.-K. Liu and B. Liu, "Expected value operator of random fuzzy variable and random fuzzy expected value models," International Journal of Uncertainty, Fuzziness and KnowlegeBased Systems, vol. 11, no. 2, pp. 195-215, 2003. 


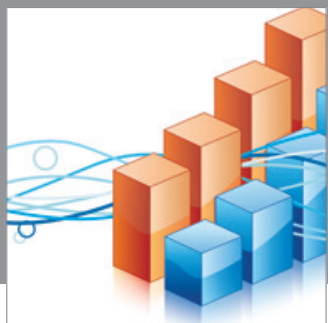

Advances in

Operations Research

mansans

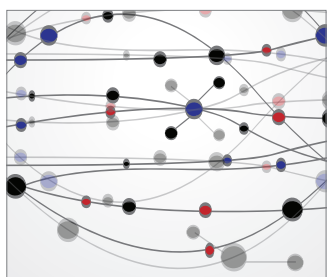

The Scientific World Journal
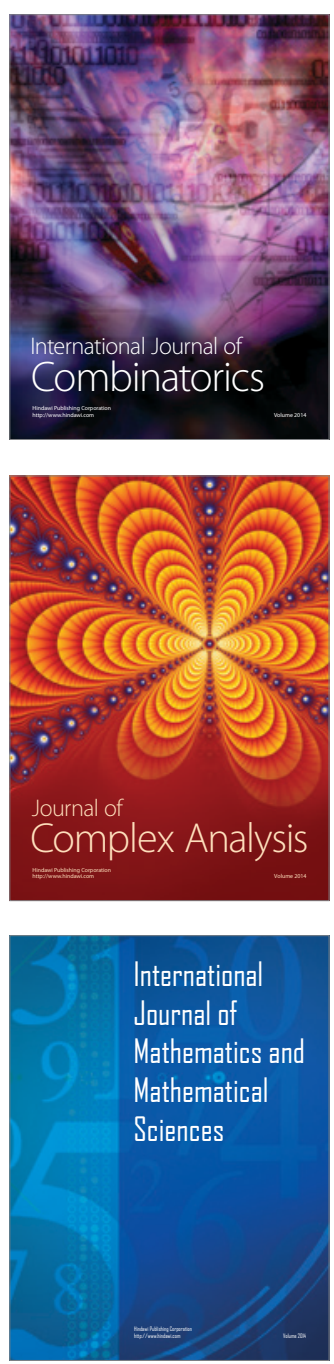
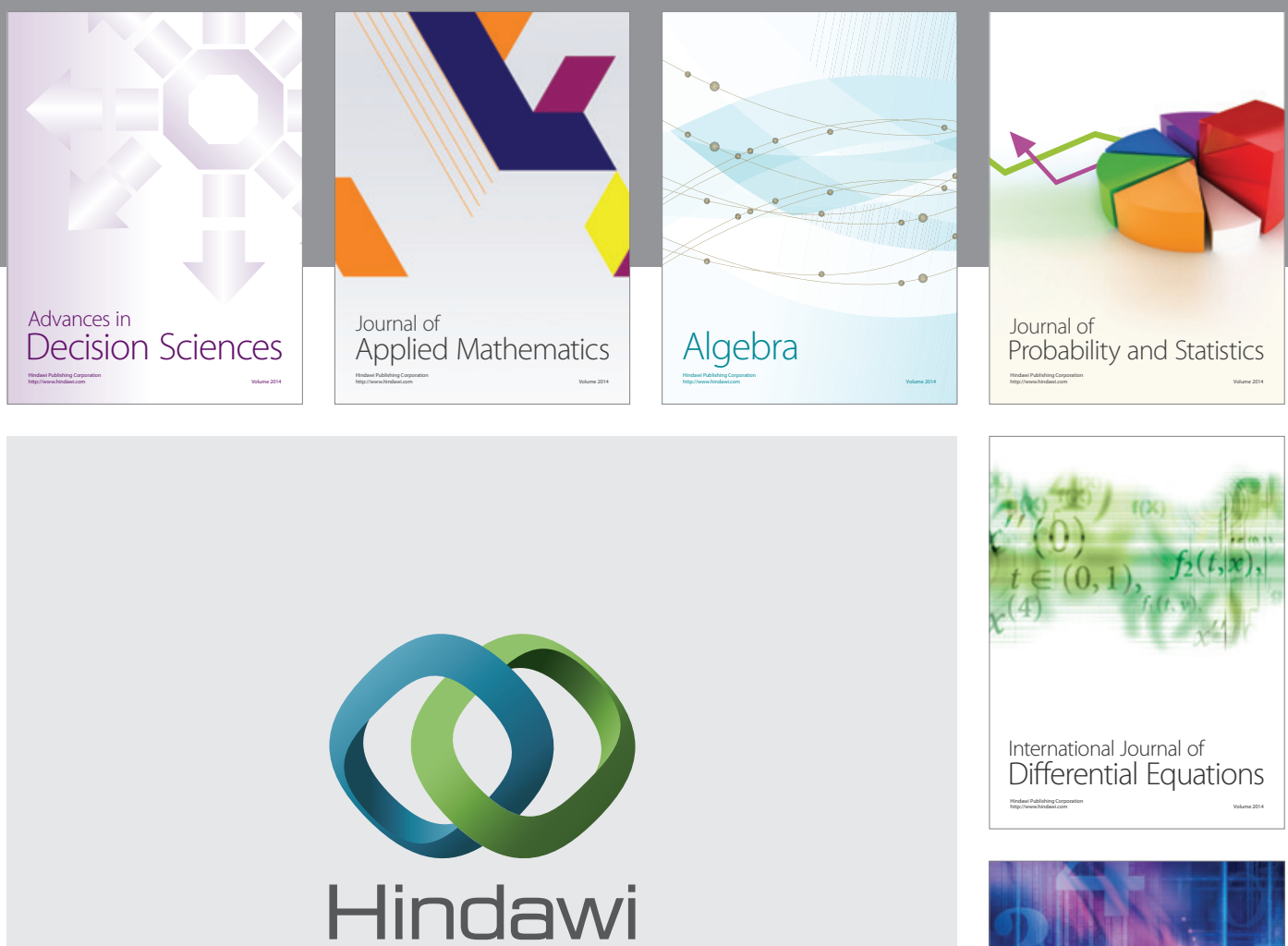

Submit your manuscripts at http://www.hindawi.com
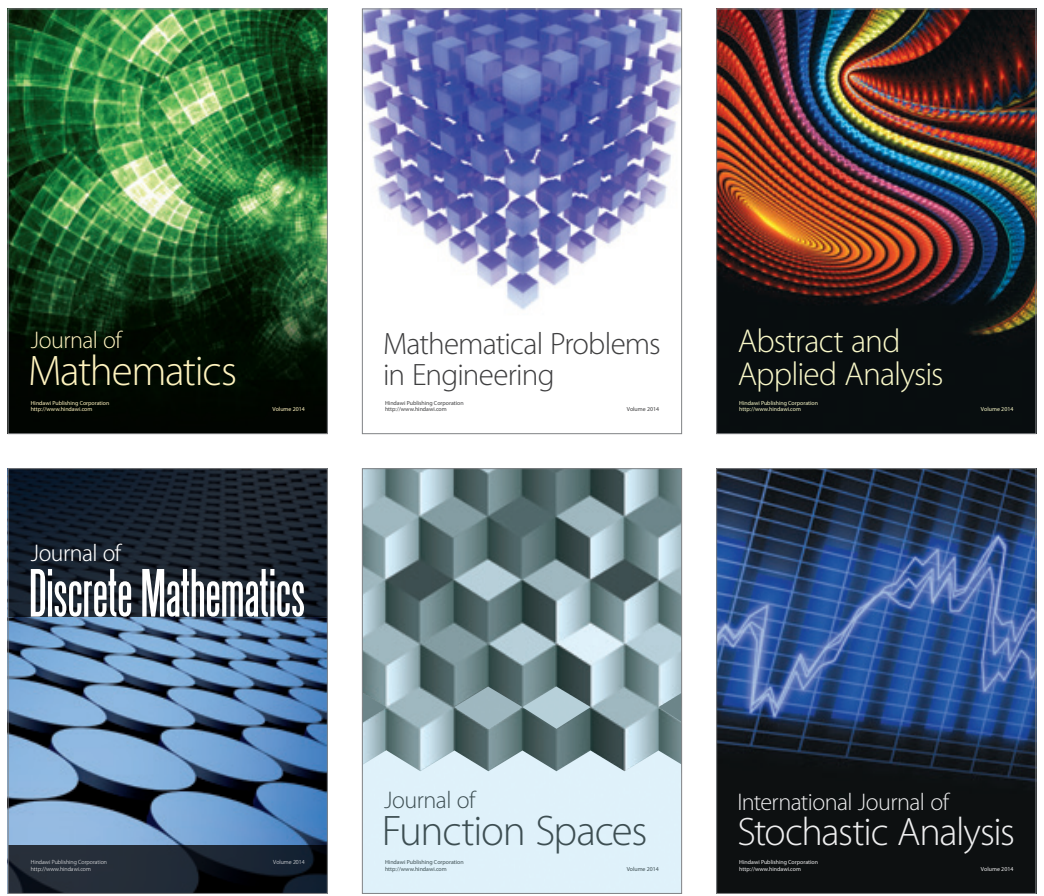

Journal of

Function Spaces

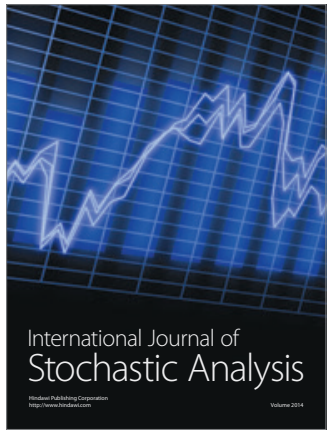

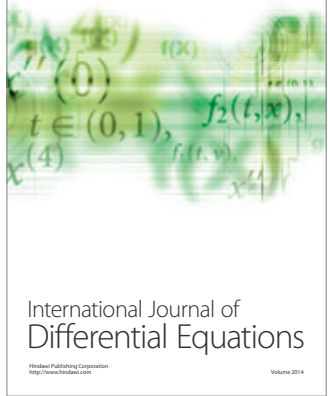
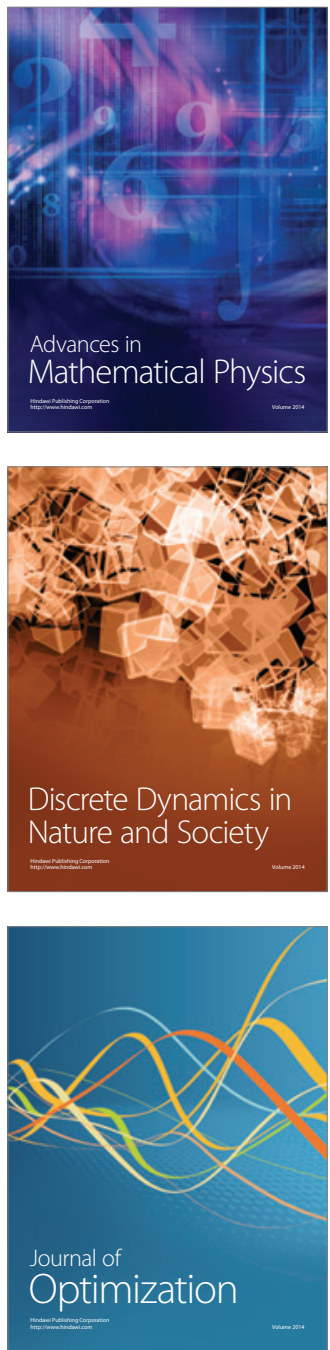